\title{
Comparison of competitive and non-competitive char formation in polymer combustion
}

\author{
S.D.WATT ${ }^{*}$, J.E.J. STAGGS*, A.C. MCINTOSH* and J. BRINDLEY ${ }^{+}$, \\ *Department of Fuel and Energy, University of Leeds, Leeds LS2 9JT, UK. \\ 'Department of Applied Mathematics, University of Leeds, Leeds LS2 9JT
}

\begin{abstract}
We consider the problem of ignition of a char-forming polymer under thermally thick conditions. In particular we explore and compare the differences in predicted ignition behaviour when models assuming competitive and non-competitive reaction schemes for char formation are used. The measure we use is the time to ignition via a critical mass flux. Finally we establish a necessary condition for the two reactions to yield different outcomes.
\end{abstract}

KEY WORDS: Ignition, char-formation, critical mass flux, modelling.

\section{INTRODUCTION}

In previous work [1], a non-competitive model of char formation for polymer ignition was discussed. The primary subject of analysis was the time taken for a polymer sample to ignite. In this work we explore the effect of modelling char formation in two different ways on predicted ignition behaviour. The non-competitive system of [1] is compared with a system involving a competitive reaction for char formation. In particular, we seek to solve the following problem. Consider a constant heating rate thermogravimetric (TG) experiment for a simple char-forming process with a single discernible step. It is possible to represent this curve using either the competitive or non-competitive models, but does the choice affect the predicted ignition behaviour under thermally thick conditions such as in a cone calorimeter experiment? In other words could any conclusions regarding the fire safety of a material be compromised by the way in which even a simple char forming process is modelled? This work represents an extension of an earlier paper [2] where the effect of subtleties of reaction mechanism on ignition was explored using a detailed dynamical systems approach under thermally thin conditions.

Our approach to this problem is simple. We consider a constant heating rate TG curve generated by a competitive reaction and fit the non-competitive model to it using a least squares method detailed in Section 4. The two different reaction schemes are then incorporated into a pyrolysis model which approximates conditions in the cone calorimeter and the ignition times for the two cases are calculated and compared. The critical mass flux criterion is used for ignition, as in the previous work [1], and which is discussed in references $[3,4]$.

In Section 5 we consider two particular cases to illustrate this approach. We also introduce a simple practical test to distinguish the two mechanisms. Basically, the 
condition is that the final char yield, for a fixed set of parameter values, will differ significantly between the two when the linear heating rate varies.

\section{PHYSICAL BASIS OF THE MODEL}

The situation we model is the combustion of a polymer in a controlled environment, for example in a cone calorimeter, where the polymer sample is heated from above. We assume that the sample is thick enough, so that there is a vertical temperature gradient, and large enough that edge effects may be neglected. The char formation chemistry is taken to be either competitive

$\mathrm{C} 1$ :

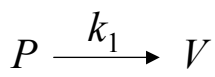

or non-competitive

C0:

$$
P \stackrel{k}{\longrightarrow} r C+(1-r) V
$$

where $P$ is polymer, $C$ is char, $V$ is volatile gas and $r$ is char yield.

\section{KINETIC REPRESENTATION OF CHAR-FORMING PROCESSES}

A constant heating rate TG curve for a simple char-forming polymer involving only a single discernible step can be modelled in various ways. The competitive and noncompetitive reaction schemes are two simple representations. In mathematical terms, the competitive model (labelled as scheme $\mathrm{C} 1$ ) is

$$
\frac{d m_{1}}{d t}=-\left\{k_{1}(T)+k_{2}(T)\right\} m_{1}, \quad \frac{d m_{2}}{d t}=k_{2}(T) m_{1}, \quad \frac{d T}{d t}=H
$$

with initial conditions

$$
m_{1}(0)=m_{0}, \quad m_{2}(0)=0, \quad T(0)=T_{a},
$$

where $m_{1}$ is the mass of virgin polymer, $m_{2}$ is the mass of char, $T$ is temperature, $t$ is time, $H$ is the constant heating rate, $m_{0}$ is the initial mass of the sample. $T_{a}$ is ambient temperature and $k_{i}=A_{i} \exp \left(-T_{A i} / T\right), i=1,2$, are reaction rates. In this model the final char yield $r$ is not known a priori and must be found by integration. In fact it is easily shown that

$$
r=\frac{1}{H} \int_{0}^{\infty} k_{2}(T) \exp \left\{-\frac{1}{H} \int_{0}^{T} k_{1}(\theta)+k_{2}(\theta) d \theta\right\} d T .
$$


The non-competitive model (labelled as scheme $\mathrm{C} 0$ ) involves only a single reaction rate $k(T)$, but an additional parameter in the form of the final char yield $r$ is explicitly added. The equations are

$$
\frac{d m_{1}}{d t}=-k(T) m_{1}, \quad \frac{d m_{2}}{d t}=r k(T) m_{1}, \quad \frac{d T}{d t}=H,
$$

with the same initial conditions :

$$
m_{1}(0)=m_{0}, \quad m_{2}(0)=0, \quad T(0)=T_{a},
$$

An earlier paper [2] considered the effect of subtleties of reaction mechanism on ignition behaviour from a dynamical systems viewpoint. In that work scheme $\mathrm{C} 1$ was considered alongside a generalised non-competitive scheme where the reactant $m_{1}$ is partitioned in such a way that a fraction $\Gamma$ of $m_{1}$ undergoes a reaction which converts it into char and $(1-\Gamma)$ undergoes an independent reaction which converts it into volatile gases. So we have

$$
\frac{d\left(\Gamma m_{1}\right)}{d t}=-k_{1}(T) \Gamma m_{1}, \quad \frac{d(1-\Gamma) m_{1}}{d t}=-k_{2}(T)(1-\Gamma) m_{1}, \quad \frac{d T}{d t}=H,
$$

from which it follows that the fraction $\Gamma$ of $m_{1}$ being converted to char at any particular time is a function of time, and can be shown to obey

$$
\frac{d \Gamma}{d t}=\Gamma(1-\Gamma)\left(k_{2}(T)-k_{1}(T)\right) .
$$

$d \Gamma / d t$ is zero for three cases : $\Gamma=0$, or $\Gamma=1$ when one only product is formed. The last case is when $k_{1}(T)=k_{2}(T)=k(T) \equiv A e^{-T_{a} / T}$. This third case is an important one since it then follows that $\Gamma=$ constant for all time and the first two equations of (6) collapse to the following :

$$
\frac{d m_{1}}{d t}=-k(T) m_{1}, \quad \frac{d m_{2}}{d t}=-\frac{d\left(\Gamma m_{1}\right)}{d t}=\Gamma k(T) m_{1} .
$$

This then is the same as scheme $\mathrm{C} 0$ above where now the fraction $\Gamma$ is the same as the final char yield $r$.

It is then possible to obtain the non-competitive scheme from the competitive by noting that the non-competitive scheme is a special case of the competitive reaction where the constants (from the competitive scheme) are assigned the values: $T_{\mathrm{A} 1}=T_{\mathrm{A}}, T_{\mathrm{A} 2}=T_{\mathrm{A}}, A_{1}=$ (1-r) $A$ and $A_{2}=r A$.

In this work, characteristic temperature $T_{c}$ and temperature range $\Delta T_{c}$ are used to characterise a single reaction rate rather than the Arrhenius parameters $T_{A}$ and $A$ as 
follows. Given a single first-order reaction with rate equation $d m / d t=-A \exp \left(-T_{A} / T\right) m$, a constant heating rate TG curve may be generated at a given heating rate $H$. This curve will have a characteristic temperature $T_{c}$ at which the mass has reduced by a factor of $c$ from its initial value. Furthermore, the characteristic temperature range over which the reduction of mass occurs is $\Delta T_{c}$. Reference [5] gives greater detail of this approach, which we summarise briefly here.

The mass of the polymer and char in both reactions can be expressed as a function of temperature instead of time. The new system for the competitive reaction is

$$
\frac{d m_{1}}{d T}=-\left(k_{1}+k_{2}\right) m_{1} / H, \frac{d m_{2}}{d T}=k_{2} m_{1} / H
$$

and for the non-competitive reaction is

$$
\frac{d m_{1}}{d T}=-k m_{1} / H, \frac{d m_{2}}{d T}=r k m_{1} / H .
$$

Given the characteristic temperature and temperature range, the activation temperature may be found by solving $P_{1}\left(T_{A} / T_{C}\right)=\Delta T_{C} \log (2) /\left(2 T_{C}\right)$, where $P_{1}(z)=1-z e^{z} E i(z)$ and $E i(z) \equiv \int_{z}^{\infty} e^{-t} t^{-1} d t$ is the exponential integral. Once $T_{A}$ is found, then the pre-exponential factor is given by $A=2 H \exp \left(T_{A} / T_{C}\right) / \Delta T_{C}$.

\section{MATCHING THE TG CURVES FOR THE COMPETITIVE AND NON- COMPETITIVE REACTIONS}

There are two ways of matching the TG curves. They are: given the competitive reaction parameters (two characteristic temperatures and temperature ranges), find the noncompetitive reactions parameters ( the characteristic temperature and temperature range and the char yield) so the curves fit, and vice versa. The option we have chosen is the former. The reason is twofold. First, as there are four competitive reaction parameters and three non-competitive reaction parameters, the latter set of parameters is unique. Second is that, as the non-competitive reaction is a special case of the competitive reaction, there is a trivial set of parameters for which the two reactions are equivalent

\subsection{The method of matching TG curves}

Assume we have a set of competitive reaction parameters $\left(T_{A 1}, T_{A 2}, \Delta T_{A 1}\right.$ and $\left.\Delta T_{A 2}\right)$. We can obtain a TG curve by solving the equations of scheme $\mathrm{C}$. The tail of the TG curve asymptotes to the final char yield. As the char yield needs to be the same in both reactions, the char yield for the non-competitive reaction, $r$, is specified. We define the characteristic temperature $T_{\mathrm{c}}$ as the temperature at which the mass of the sample is exactly halfway between the initial mass and the final char yield. That is 


$$
m\left(T_{c}\right)=m_{1}\left(T_{c}\right)+m_{2}\left(T_{c}\right)=\frac{r+1}{2}
$$

Another definition widely used is that the characteristic temperature $T_{\mathrm{c}}$ is the inflection point of the TG curve. That is $d^{2} \mathrm{~m} / d t^{2}=0$ when $T=T_{c}$. We adopt the hypothesis that the two definitions give similar results. The definition we chose simplifies the analysis. We now have two of three non-competitive reaction parameters. The only parameter left to allocate is the characteristic temperature range $\Delta T_{\mathrm{c}}$. Assuming that $T_{\mathrm{c}}$ and $r$ are now fixed, $\Delta T_{\mathrm{c}}$ must be found such that the TG curve for the non-competitive reaction is as close as possible to that of the competitive reaction. This can be achieved by a leastsquares approach. Shown in Figure 1 is a TG curve for a competitive reaction and the TG curve for a non-competitive reaction with the correct char yield and characteristic temperature, but with varying temperature ranges.

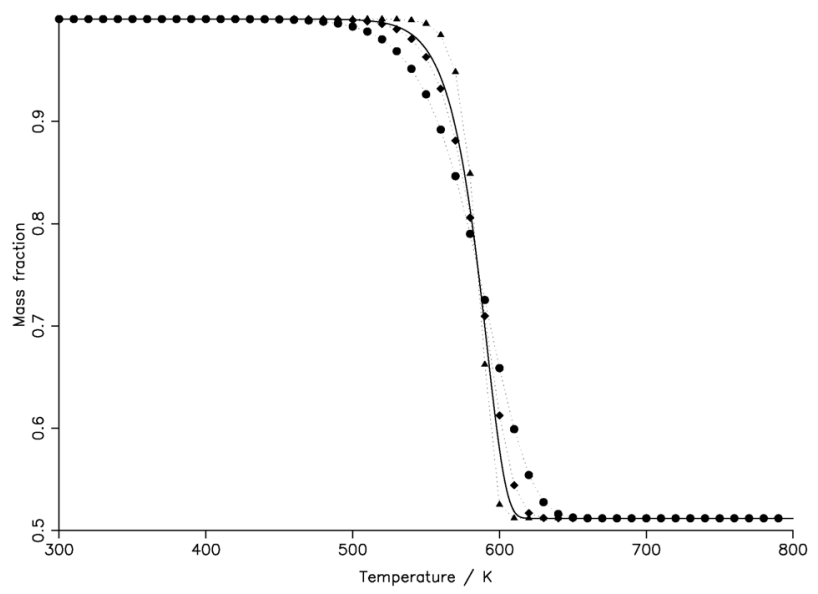

Figure 1: Thermogravimetric plot for a competitive (solid line) and noncompetitive reaction ( dashed lines).The different symbols represent different characteristic temperature ranges: $25 K$ ( triangles) $50 K$ (diamonds) and $75 K$ (circles).

\section{THE PYROLYSIS MODEL}

To model ignition under thermally thick conditions we use the pyrolysis model outlined in reference [5]. The system we investigate is drawn schematically in Figure 2, and is summarised as follows:

Polymer Degradation

$$
\frac{D \lambda}{D t}=\lambda k^{\prime}(\lambda, T)
$$


where $\lambda$ is the local mass fraction of polymer, $k^{\prime}(\lambda, T)=k_{1}(T)(\lambda-1)-k_{2}(T)$ for the competitive reaction scheme $\mathrm{C} 1$ and $k^{\prime}(\lambda, T)=k(T)\{(1-r) \lambda-1\}$ for the non-competitive scheme $\mathrm{C} 0$.

Energy equation for the sample $0 \leq y \leq s(t)$

$$
\rho c \frac{D T}{D t}=\frac{\partial}{\partial y}\left(\kappa \frac{\partial T}{\partial y}\right)-\rho Q \lambda k^{\prime \prime}(T),
$$

where $\rho$ is the density, $c$ is the specific heat capacity, $\kappa$ is thermal conductivity, $Q$ is the latent heat associated with conversion of unit mass of the virgin polymer into volatile gases and char, $k^{\prime \prime}(T)=k_{1}(T)+k_{2}(T)$ for scheme $\mathrm{C} 1$ and $k^{\prime \prime}(T)=k(T)$ for scheme $\mathrm{C} 0$.

Boundary condition on the top surface $y=s(t)$

$$
\kappa \frac{\partial T}{\partial y}=\varepsilon q+\chi\left(T_{a}-T\right)+\varepsilon \sigma\left(T_{a}^{4}-T^{4}\right),
$$

where $\varepsilon$ is the emissivity, $q$ is the external heat flux, $\chi$ is the convective heat transfer coefficient and $\sigma$ is the Stefan-Boltzmann constant.

Boundary condition on the bottom surface $y=0$

$$
\frac{\partial T}{\partial y}=0 \text {. }
$$

Initial conditions

$$
T(y, 0)=T_{a} \quad s(0)=L \quad \lambda(y, 0)=1,
$$

where $T_{a}$ is ambient temperature and $L$ is the initial thickness of the polymer.

Imposed heat flux $q$

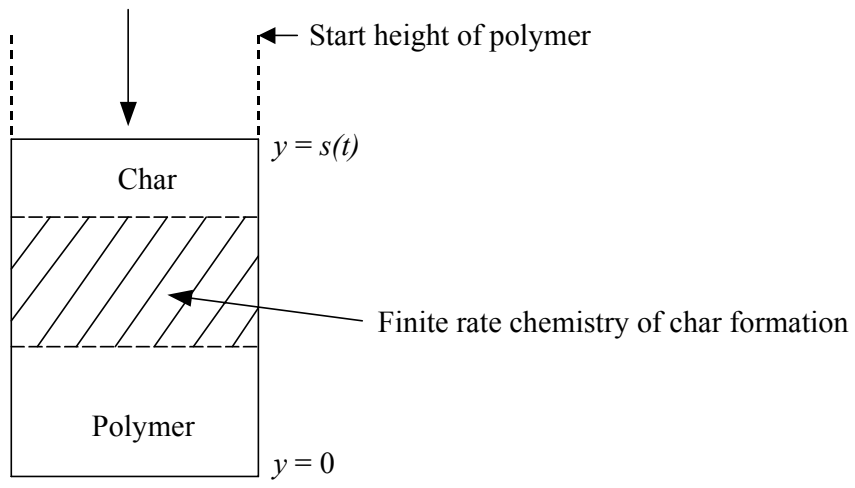

Fig. 2. Schematic of polymer degradation. 
The thermal properties are given as follows:

$$
\frac{1}{\rho}=\frac{\lambda}{\rho_{p}}+\frac{1-\lambda}{\rho_{c}}, c=c_{p} \lambda+c_{c}(1-\lambda), \kappa=\rho\left[\lambda \frac{\kappa_{p}}{\rho_{p}}+(1-\lambda) \frac{\kappa_{c}}{\rho_{c}}\right] .
$$

Here the subscript $p$ denotes a virgin polymer property and the subscript $c$ denotes a char property.

As mentioned in reference [5], the net flow of material associated with volume change induces an advection term, which means that we need to take a total derivative, given by

$$
\frac{D}{D t} \equiv \frac{\partial}{\partial t}+\left(\int_{0}^{y} \frac{\rho \lambda}{\rho_{p}} \widetilde{k}(T) d y\right) \frac{\partial}{\partial y}
$$

where $\tilde{k}(T)=k_{2}(T)(\phi-1)-k_{1}(T)$ for scheme $\mathrm{C} 1$ and $\tilde{k}(T)=k(T)(r \phi-1)$ for scheme $\mathrm{C} 0$ and $\phi=\rho_{p} / \rho_{c}$.

The regression of the top surface, $s(t)$, is then given implicitly by the ordinary differential equation

$$
\frac{d s}{d t}=\int_{o}^{s(t)} \widetilde{k} d y
$$

\section{IGNITION CRITERION}

One way of quantifying the ignition behaviour of this system is to monitor the mass flux

$$
\dot{m}^{\prime \prime}=\int_{0}^{s(t)} \rho \lambda k^{\prime \prime \prime}(T) d y,
$$

where $k^{\prime \prime \prime}(T)=k_{1}(T)$ for scheme $\mathrm{C} 1$ and $k^{\prime \prime \prime}(T)=(1-r) k(T)$ for scheme C0. The model is further discussed in reference [6] and we assume that ignition occurs as soon as the mass flux reaches a critical value, as discussed in references $[3,4]$.

\section{DIFFERENCES IN BEHAVIOUR}

It should be noted at the start that for most of the parameter space, the behaviour between a competitive and non-competitive reaction with identical TG curves does not differ much. However, there are cases where the differences are substantial, and dangerous from a fire safety viewpoint. 
The first case we consider in competitive reaction parameter space is $T_{c l}=600 \mathrm{~K}, T_{c 2}=$ $600 \mathrm{~K}, \Delta T_{c 1}=50 \mathrm{~K}$ and $\Delta T_{c 2}=25 \mathrm{~K}$. The corresponding point in non-competitive reaction parameter space is $T_{c}=585.4 \mathrm{~K}, \Delta T_{c}=41.4 \mathrm{~K}$ and $r=0.51$. Putting these parameters into the full model, we can obtain ignition times for both reactions, as a function of the external heat flux, shown in Figure 3. There are two qualitative differences. The first is that the competitive reaction has a delay in the ignition time, and takes longer to ignite. The second is that ignition occurs in the competitive model for lower external heat flux. In this case, there is no safe wrong answer. You either pick the correct reaction type or there are safety concerns.

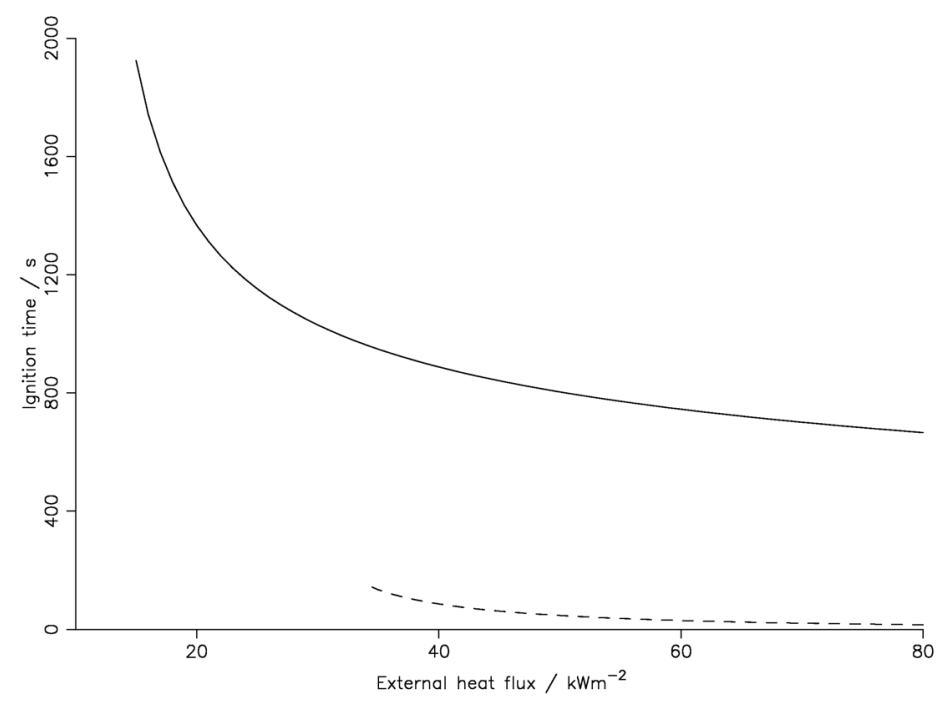

Figure 3: Plot of the ignition time for a competitive (solid line) and non-competitive reaction (dashed line).

In Figure 4, ignition times are shown for varying temperature ranges $\Delta T_{c 2}$. Note that for the purposes of the calculation, a maximum ignition time of 1800 s was chosen. It can be seen that as the temperature range increases, the ignition times agree for an increasing range of external heat flux and that ignition in the competitive reaction occurs in a decreasing range of external heat flux: the heat flux necessary to ignite the sample generally increases with increasing $\Delta T_{c 2}$. Note also the interesting behaviour arising through the development of a discontinuous jump in ignition time for cases (b) - (e). The mechanism for this arises through a combination of the ignition criterion and the pyrolysis behaviour, and the reader is referred to an earlier paper for a detailed discussion of this point [1]. Note that when $\Delta T_{c 2}=50 \mathrm{~K}$, the ignition behaviour of the competitive reaction is identical with that of the non-competitive reaction (given by the dashed curve in Figure $3)$. 


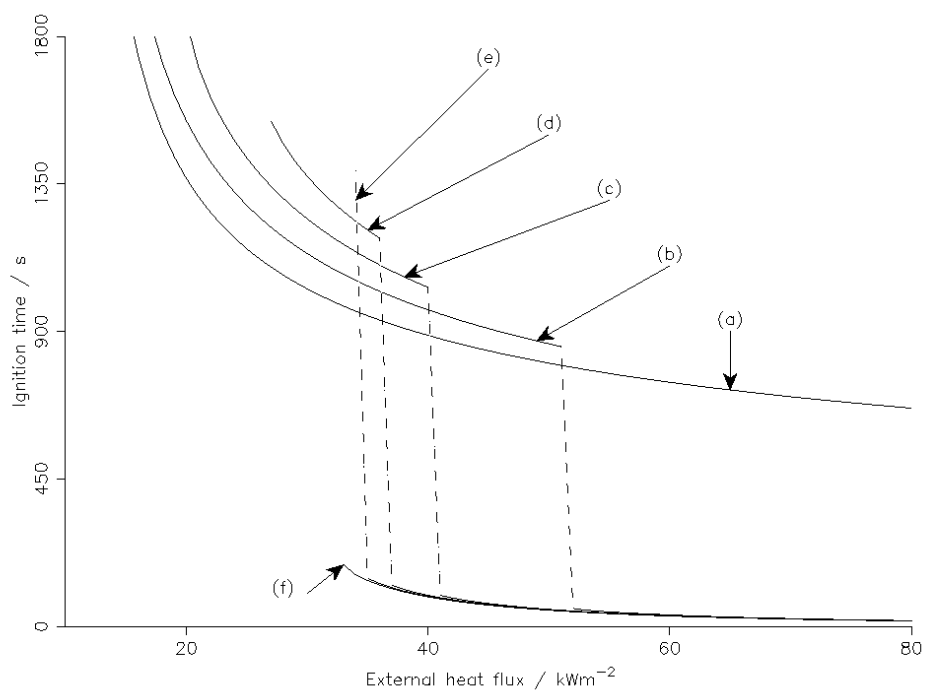

Figure 4: Plot of the ignition time for a competitive reaction with varying characteristic temperature range: (a) $25 \mathrm{~K}$ (b) $30 \mathrm{~K}$ (c) $35 \mathrm{~K}$ (d) $40 \mathrm{~K}$ (e) $45 \mathrm{~K}$ and (f) $50 \mathrm{~K}$.

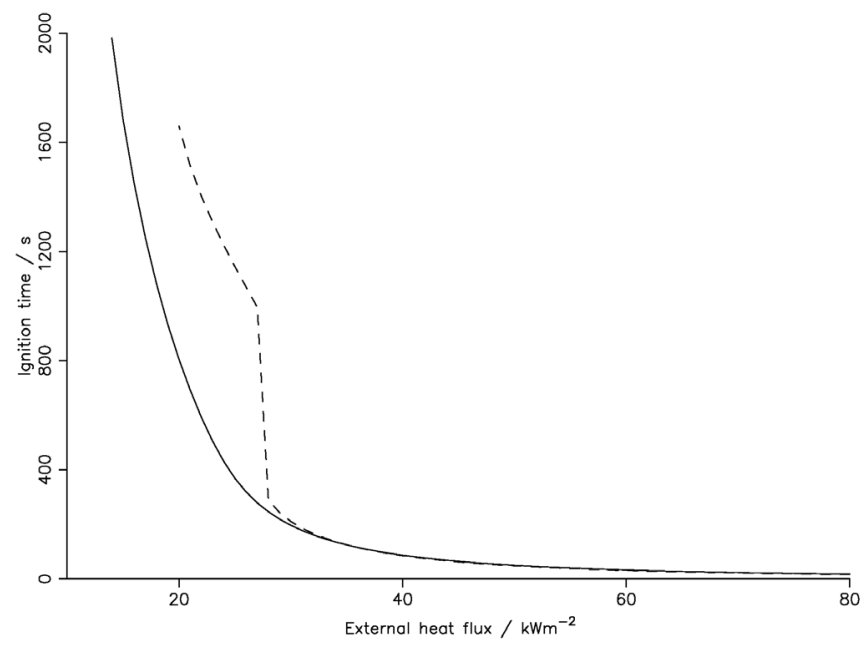

Figure 5: Plot of the ignition time for a competitive (solid line) and non-competitive reaction (dashed line).

The second case we consider in competitive reaction parameter space is $T_{c l}=600 \mathrm{~K}, T_{c 2}=$ $610 \mathrm{~K}, \Delta T_{c 1}=50 \mathrm{~K}$ and $\Delta T_{c 2}=20 \mathrm{~K}$. The corresponding point in non-competitive reaction parameter space is $T_{c}=591.3 \mathrm{~K}, \Delta T_{c}=42.5 \mathrm{~K}$ and $r=0.32$. This case differs from the first, as the non-competitive reaction has a discontinuous jump in the ignition 
time, whereas the competitive reaction does not. This is shown in Figure 5. As the temperature range $\Delta T_{c 2}$ is increased, then a jump of ignition time occurs in the competitive reaction, as shown in Figure 6 (where, as in Figure 4, a maximum ignition time of 1800s was chosen). Note also from Figure 6 that the ignition behaviour becomes insensitive to the variation in $\Delta T_{c 2}$ as heat flux increases, indicating that ignition behaviour is being dominated by the volatile-forming step (with lower characteristic temperature).

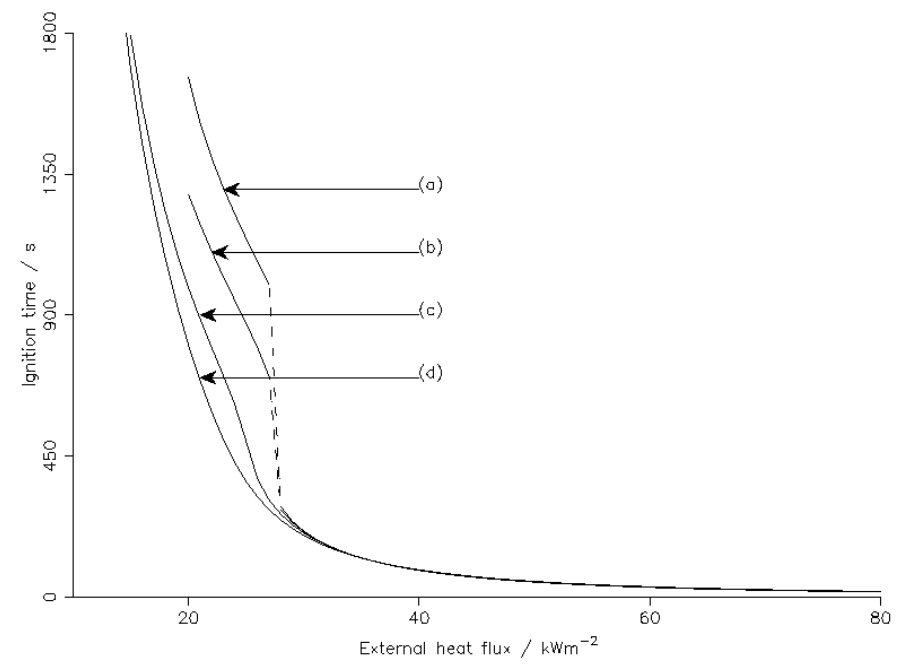

Figure 6: Plot of the ignition time for a competitive reaction with varying characteristic temperature range of the char-forming step $\Delta T_{\mathrm{c} 2}$ : (a) $20 \mathrm{~K}$ (b) $30 \mathrm{~K}$ ( c) $40 \mathrm{~K}$ and (d) $50 \mathrm{~K}$.

A necessary condition for there to be a substantial difference in the ignition behaviour is that the char yield in the competitive reaction be sensitive to the heating rate $H$. This can be found by varying the heating rate in the rate equations (1), but keeping the Arrhenius parameters fixed. From the generated TG curves, the final char yield can be taken as the asymptote of this curve. In Figure 7, the final char yield has been calculated for $T_{c l}=600$ $\mathrm{K}, T_{c 2}=610 \mathrm{~K}, \Delta T_{c l}=50 \mathrm{~K}$ and varying $\Delta T_{c 2}$. What can be seen is that as the temperature range increases, the variation of the final char yield decreases, until $\Delta T_{c 2}=50 \mathrm{~K}$ where the final char yield is $50 \%$ regardless of the heating rate. The characteristic temperature ranges used in the non-competitive reaction is that marked on the right hand end of each of these curves.

An experimental method to demonstrate this condition might be to heat the sample at the original heating rate and then at twice that value. This will give two final char yields. The larger the difference between the two, the greater the chance that the polymer is best modelled by the competitive model, and when the difference is small, the polymer is best modelled by a non-competitive model. 


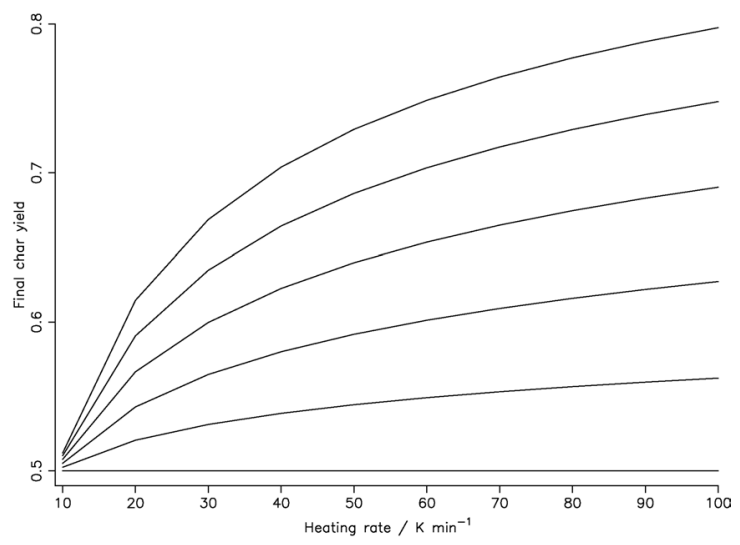

Figure 7: Plot of the final char yield for varying heating rates and characteristic temperature ranges $\Delta T_{\mathrm{c} 2}$.

In Figure 8, we have made a contour plot of these differences for $T_{c l}=600 \mathrm{~K}$ and $\Delta T_{c l}=$ $50 \mathrm{~K}$. From this, we would expect a larger qualitative difference between the two schemes, the nearer the parameters were to the set $T_{c 2}=610 \mathrm{~K}$ and $\Delta T_{c 2}=25 \mathrm{~K}$ or $T_{c 2}=$ $580 \mathrm{~K}$ and $\Delta T_{c 2}=75 \mathrm{~K}$.

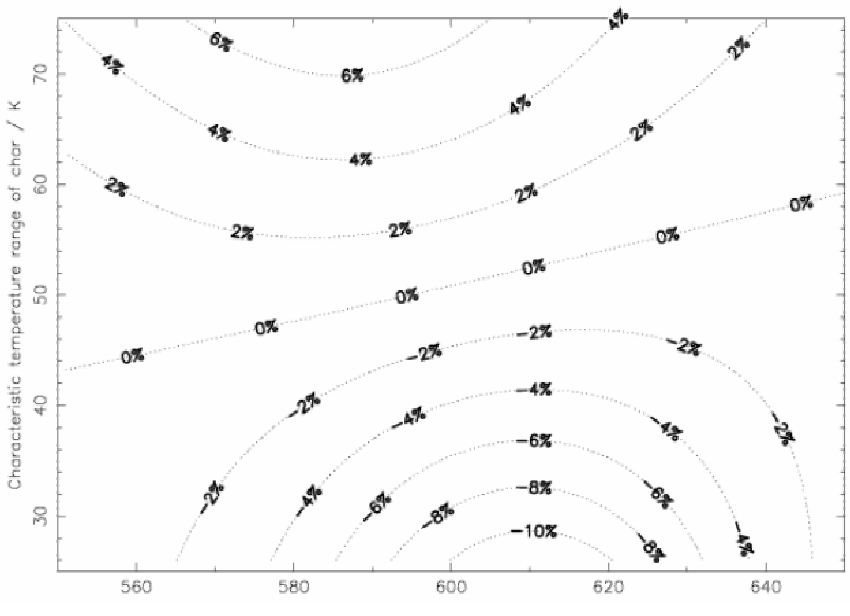

Characteristic temperature. of char $/ \mathrm{K}$

Figure 8: Contour plot of the difference in final char yield at $10 \mathrm{~K} / \mathrm{min}$ and $20 \mathrm{~K} / \mathrm{min}$ 


\section{CONCLUSION}

We have considered the importance of the choice of competitive or non-competitive reaction models for the char formation in the ignition of a polymer sample. We have deliberately not included experimental comparisons because the main thrust of the work is to examine the effect of different theoretical models on predicted ignition behaviour. We have outlined a method by which, for a given set of competitive reaction parameters, a set of non-competitive reaction parameters can be found, such that the TG curves are identical. By looking through parameter space, we can find cases where there are qualitative differences in the ignition behaviour of the two reactions. However, it should be noted that most of time, there are no large differences. Despite this, the result is important and points to the fact that the analysis of the combustion of a char forming polymer needs to be classified by more than a TG curve.

\section{ACKNOWLEDGEMENT}

This work was carried out whilst SDW was supported by the EPSRC (GR/L28142).

\section{REFERENCES}

[1] S.D. Watt, J.E.J. Staggs, A.C. Mclntosh and J. Brindley "A theoretical explanation of the influence of the char formation on the ignition of polymers" Fire Safety Journal $\underline{36}$, 421-436 (2001).

[2] R. Ball, A.C. Mclntosh and J. Brindley "Thermokinetic models for simultaneous reactions: a comparative study" Combust. Theory \& Modelling 3 pp. 447-468 (1999).

[3] Nelson, M.I., Brindley, J. and McIntosh, A.C. "The dependence of critical heat flux on fuel and additive properties : a critical mass flux model", Fire Safety Journal 24, 107130 (1995).

[4] Staggs J. E. J. \& Nelson M. I. "A critical mass flux model for the flammability of thermoplastics". Combust. Theory Modelling 5 pp399-427 (2001).

[5] J. E. J. Staggs. "Modelling thermal degradation of polymers using single-step firstorder kinetics". Fire Safety Journal 32 pp17-34 (1999).

[6] J.E.J .Staggs "Simple Mathematical Models of Char- Forming Polymers" Polymer International 49 pp1147-1152 (2000). 